Urologe 2013 · 52:479-480

DOI 10.1007/s00120-012-3093-1

Online publiziert: 20. März 2013

(c) Springer-Verlag Berlin Heidelberg 2013

R.M. Bauer · C.G. Stief

Urologische Klinik und Poliklinik, Klinikum der Universität München-Großhadern, München

\title{
Urologische Bildgebung: Was geht in 2013
}

ten Biopsieverfahren bei der Diagnostik des Prostatakarzinoms sowie die aktuelle Datenlage. Sie zeigen dabei auf, dass eine Biopsie mit Hilfe der MRT-TRUS-Fusionsbildgebung v. a. bei Männern mit bereits erfolgter negativer Biopsie sinnvoll erscheint, aber aktuell v. a. noch technische Probleme eine Etablierung in den klinischen Alltag verhindert.

Verschiedene moderne bildgebende Verfahren wie Histoscanning, ANNA/CTRUS, Elastographie und Kontrastmittelultraschall, zur Darstellung der Prostata selber werden von Jochen Walz und Kollegen dargestellt und verglichen. Dabei ziehen die Kollegen abschließend das Resümee, dass bisher keine multizentrischen Studien für sämtliche zur Verfügung stehenden Bildgebungsverfahren existieren, aber diese für eine Etablierung in den klinischen Alltag dringend notwendig sind.

Alexander Kretschmer und Kollegen gehen der Frage nach, welche Bildgebung beim fortgeschrittenen Prostatakarzinom sinnvoll ist und zeigen, dass sich aufgrund diverser technischer Weiterentwicklungen und Innovationen die Bildgebung des fortgeschrittenen Prostatakarzinoms im Wandel befindet und mittlerweile das Cholin-PET im klinischen Alltag eine wichtige Rolle spielt. Die Kollegen weisen aber auch darauf hin, dass nicht „Alles was machbar ist" auch sinnvoll ist. Kritisch merken sie allerdings an, dass ,genaueres Wissen“ oftmals die Prognose der Patienten nicht verbessert.

Die Arbeitsgruppe um Alexander Karl beschäftigt sich mit der Indikation der photodynamischen Diagnostik beim Blasentumor und stellt aktuelle Studienergebnisse dar. Darüber hinaus wird auch auf den Stellenwert der ambulanten PDD-
Diagnostik mittels flexiblen Zystoskops hingewiesen, die heute mittels der hochmodernen „Chip-on-the-tip-Technologie" prinzipiell technisch machbar wurde.

Tobias Maurer und Kollegen diskutieren die Wertigkeit der funktionellen Bildgebung mittels PET/CT und MRT beim Blasenkarzinom. Die konventionelle CT stellt zwar die aktuelle präoperative Standardbildgebung dar, hat aber v. a. Schwächen hinsichtlich des lokalen Tumor- und Lymphknotenstagings. Moderne MRTSpezialsequenzen als auch die ${ }^{18}$ F-FDGPET/CT scheinen hier zwar Vorteile zu bieten, die aktuelle Studienlage ist aber zu schwach, um diesbezüglich schon eine generelle Empfehlung aussprechen zu können.

Dirk Clevert und seine Arbeitsgruppe stellen das Konzept der multimodalen Diagnostik bei unklaren Raumforderungen der Niere vor. Dabei beantworten sie die Fragen wie gut der Kontrastmittelultraschall ist und welche Rolle die CT bei der Diagnostik von Nierenraumforderungen spiel. Darüber hinaus wird dargestellt, wann das MRT zur Diagnostik bei Nierenraumforderungen eingesetzt werde sollte. Für den klinischen Alltag wird ein alltagstauglicher Leitfaden für die Wahl der jeweiligen optimalen diagnostischen Methode dargestellt.

Ruth Kirschner-Hermanns und Kollegen diskutieren die Aussagekraft und Nutzen der verschiedenen bildgebenden Verfahren zur Diagnostik der männlichen Harninkontinenz, die sich im Vergleich zu der bildgebenden Diagnostik, insbesondere mittels Ultraschall bei der Frau, noch wenig durchgesetzt haben. Vor allem die perineale Sonographie erscheint hier für den klinischen Alltag geeignet, da
David Schilling und Kollegen diskutieren den aktuellen Stand der MRT-gestütz- 
insbesondere auch die sog. "male slings“ gut dargestellt werden können. Das MRT und dabei insbesondere das dynamische Funktions-MRT bleiben eher der klinischen Forschung und speziellen Fragestellungen, wie unklaren Komplikationen nach „male slings“ vorbehalten.

Den Stellenwert der Sonographie bei der Diagnostik von Funktionsstörungen des weiblichen Beckenbodens erörtern Stefan Albrich und Kollegen. Im Alltag der Urogynäkologie ist heute der Ultraschall am Beckenboden zu einem unverzichtbaren Bestandteil der Routinediagnostik geworden. Er ist dabei nicht nur äußerst wertvoll bei der subtilen Diagnostik sondern auch bei der postoperativen Darstellung von alloplastischen Implantaten.

Frank Strittmatter und Kollegen stellen die modernen bildgebenden Verfahren bei der Diagnostik der Urolithiasis dar und diskutieren die Vor- und Nachteile der vorhandenen Methoden. Darüber hinaus zeigen sie, dass mit der „Dualenergy-CT“ ein innovatives Verfahren zur Verfügung steht, bei dem nicht nur die Steinlokalisation und -größe bestimmt, sondern auch mit hoher Sicherheit Auskunft über die Steinzusammensetzung (die einen wesentlichen Einfluss auf die weitere Therapiestrategie hat) gegeben werden kann.

Damit die Urologie auch weiterhin ein hoch innovatives Fach nicht nur in Bezug auf Therapiemöglichkeiten, sondern auch im diagnostischen Bereich bleibt, ist eine weitere intensive Forschungstätigkeit in diesem Bereich notwendig. Nur wenn wir an der Spitze der Innovationen stehen und diese (mit)gestalten und vorantreiben, werden wir weiter als Fach existieren.

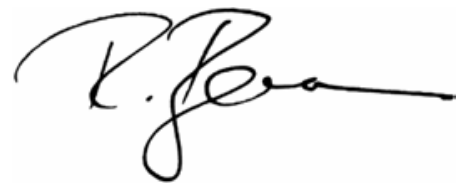

R.M. Bauer

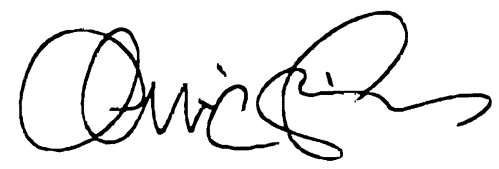

C.G. Stief

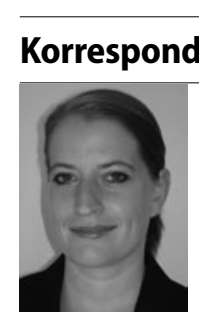

\section{Medizin-Management-Preis 2013 ausgeschrieben}

PD Dr. R.M. Bauer

Urologische Klinik und Poliklinik,

Klinikum der Universität

München-Großhadern,

Marchioninistraße 15,

81377 München

Ricarda.Bauer@

med.uni-muenchen.de

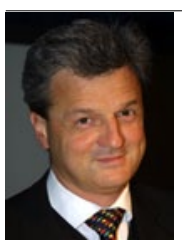

Prof. Dr. C.G. Stief

Urologische Klinik und

Poliklinik,

Klinikum der Universität

München-Großhadern,

Marchioninistraße 15,

81377 München

Christian.stief@

med.uni-muenchen.de
Unter der Schirmherrschaft des Bundesforschungsministers a. D., Herrn Professor Dr. Heinz Riesenhuber, wurde der Medizin-Management-Preis 2013 ausgeschrieben. Der Preis zeichnet Innovationen aus, die durch neue Wege des Steuerns, Führens und Leitens in der Gesundheitsbranche Ergebnisse verbessern.

Als Preisträger in Frage kommen Projekte und Konzepte jeglicher Segmente des Medizinmarktes:

- Forschung

- Verfahren

- Versorgungs- und Kooperationsformen

- Qualitätssicherung und Produktion

- Informationstechnologie (IT)

- Kommunikation

- Human Resources

Der jährlich vergebene Preis ist mit 2.000 Euro dotiert. Bewerbungsschluss ist der

31. Mai 2013. Die Teilnahme am Wettbewerb ist kostenfrei.

Die Preisverleihung findet am 6. September 2013 in Berlin statt.

Rückblick: Im vergangenen Jahr ging der Medizin-Management-Preis an das Forensische Online-Konsil „Forensikon“ der Medizinischen Hochschule Hannover (MHH). Dieses ermöglicht Ärzten, bei Verdachtsfällen von Kindesmissbrauch und Kindesmisshandlung fragliche Befunde zeitnah und ohne Umweg über Behörden durch Rechtsmediziner beurteilen zu lassen. Ausführliche Informationen zum Preis, dem Bewerbungsverfahren und den prämierten Innovationen der vergangenen Jahre unter: www.medizin-management-preis.de

Quelle: Medizin-Management-Verband e.V. (Hamburg). 